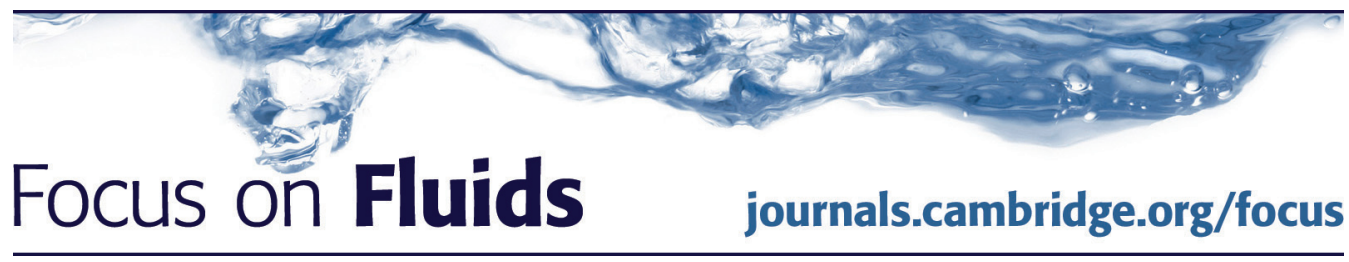

\title{
The fate of random initial vorticity distributions for two-dimensional Euler equations on a sphere
}

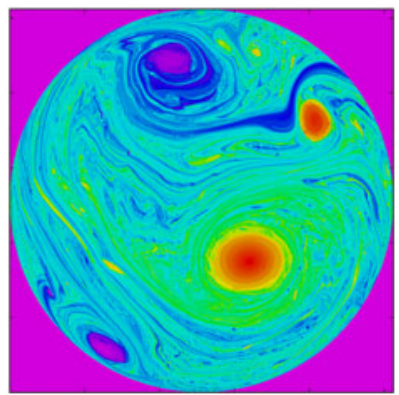

\section{Paul K. Newton $\dagger$}

Department of Aerospace and Mechanical Engineering and Mathematics, University of Southern California,

Los Angeles, CA 90089-1191, USA

The paper by Dritschel et al. (J. Fluid Mech., vol. 783, 2015, pp. 1-22) describes the long-time behaviour of inviscid two-dimensional fluid dynamics on the surface of a sphere. At issue is whether the flow settles down to an equilibrium or whether, for generic (random) initial conditions, the long-time solution is periodic, quasi-periodic or chaotic. While it might be surprising that this issue is not settled in the literature, it is important to keep in mind that the Euler equations form a dissipationless Hamiltonian system, hence the set of equations only redistributes the initial vorticity, generating smaller and smaller scales, while keeping kinetic energy, angular impulse and an infinite family of vorticity moments (Casimirs) intact. While special solutions that never settle down to an equilibrium state can be constructed using point vortices, vortex patches and other distributions, the fate of random initial conditions is a trickier problem. Previous statistical theories indicate that the long-time state should be a stationary large-scale distribution of vorticity. By carrying out careful numerical simulations using two different methods, the authors make a compelling case that the generic long-time state resembles a large-scale oscillating quadrupolar vorticity field, surrounded by persistent small-scale vortices. While numerical simulations can never conclusively settle this issue, the results might help guide future theories that seek to prove the existence of such an interesting dynamical long-time state.

Key words: atmospheric flows, low-dimensional models, vortex dynamics

\section{Introduction}

On 23 August 2005, a concentrated vorticity region began to form off the south coast of Florida over the ocean, reaching its peak strength on 28 August, touching land over the city of New Orleans and parts of the Florida coast. Clouds and moisture

$\dagger$ Email address for correspondence: newton@usc.edu 
advected by the vorticity field act as tracers for the flow, making Hurricane Katrina visible to the human eye, as seen in http://sos.noaa.gov/Datasets/dataset.php?id=490. This category 5 hurricane, the fifth hurricane of the 2005 Atlantic hurricane season, was the third most intense ever to hit continental USA, and caused roughly 1800 fatalities and $\$ 100$ billion in damage before dissipating on 31 August.

Given the evident intense concentration of vorticity in one localized region on the surface of the globe for this terrifying week, two natural questions arise: (1) How did that particular vorticity distribution arise at that particular location when it did? and (2) What will be the future evolution of that vorticity distribution, one day, one week, one month, or one year ahead? The first question (genesis) is typically the focus of atmospheric scientists and meteorologists who study hurricane theory (see Emanuel 1991). These studies focus on combined models involving fluid mechanics on a stratified and rotating sphere, thermodynamics and atmospheric and oceanographic processes. The equations and models are complex (Emanuel 1991) and simulations are run on dedicated supercomputers at the National Center for Atmospheric Research (NCAR) in Boulder, Colorado, and elsewhere. Since Charney's landmark paper on numerical simulations of atmospheric flows (Charney 1949), the field of numerical weather prediction and computational atmospherics has blossomed into a major branch of computational fluid dynamics. This is because of its great relevance to important generic structures, such as hurricanes (Emanuel 1991), Jupiter's Great Red Spot (Marcus 1993), and events such as the polar vortex splitting event (Juckes \& McIntyre 1987; Charlton et al. 2005).

Applied mathematicians and theoretical fluid dynamicists tend to focus more on the second question (initial value problems) using stripped down models with (more complex) features added only after a thorough understanding is achieved in the simpler setting. From this hierarchical vantage point, it is generally agreed that two of the main features making the dynamics complex are present in the simplest stripped down Euler equations for incompressible flow on the sphere:

$$
\begin{gathered}
\boldsymbol{u}_{t}+\boldsymbol{u} \cdot \nabla \boldsymbol{u}=-\nabla p, \\
\nabla \cdot \boldsymbol{u}=0, \\
\boldsymbol{u}(\boldsymbol{x}, 0)=\boldsymbol{f}(\boldsymbol{x}) .
\end{gathered}
$$

Here, $\boldsymbol{u}$ is the fluid velocity field, $\nabla p$ is the pressure gradient (for unit density without loss of generality). The two main terms of (1.1) which make the subject of incompressible fluid mechanics interesting are (i) the nonlinear inertial term $\boldsymbol{u} \cdot \nabla \boldsymbol{u}$, and (ii) the pressure gradient term $\nabla p$, acting simultaneously in a dynamical setting, with initial conditions for the velocity field (1.3). The advective nonlinearity is generally responsible for the associated small-scale cascade after sufficiently long times. It is more direct, of course, to work with the vorticity field $\omega(\boldsymbol{x}, t) \equiv \boldsymbol{\nabla} \times \boldsymbol{u}$ than the velocity field. It is also common to consider the atmosphere as a two-dimensional spherical shell surrounding the Earth, since the vertical length scales are much smaller than horizontal scales, and because of the additional 'flattening' of the vertical scales due to stratification.

\section{Overview}

With viscous dissipation (high-Reynolds-number flows) balanced by forcing, we know that a turbulent $-5 / 3$ spectrum is generically obtained in simple settings like one-dimensional tidal channels (Grant, Stewart \& Moilliet 1962), although with 
complex vorticity distributions and in doubly periodic or spherical domains this clean scaling law has been questioned (Fontane, Dritschel \& Scott 2013). When the viscosity is reduced little by little (i.e. increasing Reynolds number) and, in tandem, the forcing is reduced little by little, the long-time fate of generic initial conditions, energy transfer and the turbulent spectrum is also well understood. But in the limit when the viscosity is zero and there is no forcing, as in the case of the Euler equations, all bets are off as to the long-time dynamics, particularly if one is interested in what are the generic 'low-dimensional' structures that dominate the vorticity field. In this delicate limit, kinetic energy, angular momentum and an infinite family of vortex moments $\left(\int \omega^{n} \mathrm{~d} S\right)$ are conserved, which provide serious constraints on the flow and its evolution. These constraints have the potential to maintain persistent coherent structures in the flow field.

Special solutions that represent mostly geophysically relevant configurations have been constructed via point vortex approximations (see Kidambi \& Newton 1998; Newton 2001, 2010; Jamaloodeen \& Newton 2006; Newton \& Shokraneh 2006; Chamoun, Kanso \& Newton 2009), distributed vorticity fields (Crowdy 2004; Newton \& Sakajo 2007), and careful numerical simulations (Tang \& Orszag 1978; Dritschel \& Polvani 1992; Marcus 1993; Polvani \& Dritschel 1993). General questions have been posed regarding generic structures that emerge (Dritschel 1993) and we refer the reader to discussions and references to the literature in the broad introduction to the topic provided by Dritschel, Qi \& Marston (2015).

Previous lab work and numerical experiments often show that initial conditions evolve to steady large-scale circulation patterns that dominate the flow field, but it is hard to rule out the influence of an ever present small amount of viscosity in the flow, which after long times can have a significant influence on the structures that emerge. In doubly periodic domains, unsteady long-time states have been shown to exist, but it is hard to rule out the possibility that the simulations were not carried out long enough in time and with high enough resolution. After all, with an infinite number of vorticity invariants to conserve and the flow generating smaller and smaller scales, the precision necessary to accurately recreate events always becomes an issue. It is generally believed that if the flow undergoes strong turbulent mixing during evolution, it will settle to a steady state. Statistical theories can explain this result, but difficulties always arise in verifying these theories due to the infinite number of conserved vorticity moments and the finite numerical precision. Issues also arise as to whether generic 2D flows are ergodic, which statistical theories assume. Ergodicity is notoriously difficult to verify numerically.

Dritschel et al. (2015) offer a plausible alternative to a long-time steady state. These authors assert that the long-time solution of the Euler equations on the sphere is not an equilibrium configuration, but a much more complex dynamical state. The state more resembles a quadrupolar large-scale oscillating vorticity state, surrounded by a sea of smaller-scale vortices that are more or less advected by the larger-scale field. Most simply, the quadrupolar vorticity field can be modelled (i.e. approximated) by an $N$-vortex configuration on the sphere, where $N=4$. In this state, the point vortices generically move chaotically or quasi-periodically (see Newton 2001).

\section{Future}

So, while Dritschel et al. (2015) cannot (and do not) claim that the issue is now fully settled, there is a new candidate generic dynamical state that had not previously been identified for the two-dimensional Euler equations on the sphere: a quasi-periodic 
or chaotic configuration dominated by a quadrupolar structure surrounded by a sea of smaller-scale vortices. What remains, aside from verification via still higher-resolution and longer numerical simulations, is a comprehensive theory that would predict such a state a priori. It is certainly hard to imagine that such a theory could have been developed before this detailed and high-quality analysis identified the candidate longtime state. From that point of view, this result offers the exciting beginnings of a plausible alternative to a steady-state theory and should be of great interest to the community.

\section{References}

Chamoun, G., Kanso, E. \& Newton, P. K. 2009 von Kármán vortex streets on the sphere. Phys. Fluids 21, 116603.

Charlton, A. J., O’Neill, A., Lahoz, W. A. \& Berrisford, P. 2005 The splitting of the stratospheric polar vortex in the southern hemisphere, September 2002: Dynamical Evolution. J. Atmos. Sci. 62, 590-602.

CHARNEY, J. 1949 On a physical basis for numerical prediction of large-scale motions in the atmosphere. J. Meteorol. 6, 371-385.

Crowdy, D. G. 2004 Stuart vortices on a sphere. J. Fluid Mech. 498, 381-402.

Dritschel, D. G. 1993 Vortex properties of two-dimensional turbulence. Phys. Fluids A 5, 984-997.

Dritschel, D. G. \& Polvani, L. M. 1992 The roll-up of vorticity strips on the surface of a sphere. J. Fluid Mech. 234, 47-69.

Dritschel, D. G., QI, W. \& MARston, J. B. 2015 On the late-time behavior of a bounded, inviscid two-dimensional flow. J. Fluid Mech. 783, 1-22.

Emanuel, K. A. 1991 The theory of hurricanes. Annu. Rev. Fluid Mech. 23, 179-196.

Fontane, J., Dritschel, D. G. \& Scott, R. K. 2013 Vortical control of forced two-dimensional turbulence. Phys. Fluids 25, 015101.

Grant, H. L., Stewart, R. W. \& Moilliet, A. 1962 Turbulent spectra from a tidal channel. J. Fluid Mech. 12, 241-268.

Jamaloodeen, M. I. \& Newton, P. K. 2006 The $N$-vortex problem on a rotating sphere. II Heterogeneous Platonic solid equilibria. Proc. R. Soc. Lond. A 462 (2065), 3277-3299.

Juckes, M. N. \& MCInTYRe, M. E. 1987 A high-resolution one-layer model of breaking planetary waves in the stratosphere. Nature 328, 590-596.

Kidambi, R. \& Newton, P. K. 1998 Motion of three point vortices on a sphere. Physica D 116 (1-2), 143-175.

Marcus, P. S. 1993 Jupiter's Great Red Spot and other vortices. Annu. Rev. Astron. Astrophys. 31, 523-573.

Newton, P. K. 2001 The N-Vortex Problem: Analytical Techniques, Applied Mathematics Science, vol. 101. Springer.

Newton, P. K. 2010 The $N$-vortex problem on a sphere: Geophysical mechanisms that break integrability. Theor. Comput. Fluid Mech. 24, 137-149.

Newton, P. K. \& Shokraneh, H. 2006 The $N$-vortex problem on a rotating sphere. I Multifrequency configurations. Proc. R. Soc. Lond. A 462 (2065), 149-169.

Newton, P. K. \& SAKajo, T. 2007 The $N$-vortex problem on a rotating sphere. III Ring configurations coupled to a background field. Proc. R. Soc. Lond. A 463, 961-977.

Polvani, L. M. \& Dritschel, D. G. 1993 Wave and vortex dynamics on the surface of a sphere. J. Fluid Mech. 255, 35-64.

TANG, C. M. \& ORSZAG, S. A. 1978 Two-dimensional turbulence on the surface of a sphere. J. Fluid Mech. 87 (2), 305-319. 